\title{
ARTICLE
}

Clinical Study

\section{Efficacy and safety of pembrolizumab in recurrent/metastatic head and neck squamous cell carcinoma: pooled analyses after long-term follow-up in KEYNOTE-012}

Ranee Mehra ${ }^{1,17}$, Tanguy Y. Seiwert ${ }^{2}$, Shilpa Gupta ${ }^{3,18}$, Jared Weiss ${ }^{4}$, Iris Gluck ${ }^{5}$, Joseph P. Eder ${ }^{6}$, Barbara Burtness ${ }^{1,19}$, Makoto Tahara7, Bhumsuk Keam ${ }^{8}$, Hyunseok Kang ${ }^{9}$, Kei Muro ${ }^{10}$, Ravit Geva ${ }^{11}$, Hyun Cheol Chung ${ }^{12}$, Chia-Chi Lin ${ }^{13}$, Deepti Aurora-Garg ${ }^{14}$, Archana Ray ${ }^{14}$, Kumudu Pathiraja ${ }^{14}$, Jonathan Cheng ${ }^{14}$, Laura Q. M. Chow ${ }^{15}$ and Robert Haddad ${ }^{16}$

BACKGROUND: Second-line treatment options for advanced head and neck squamous cell carcinoma (HNSCC) are limited. The phase Ib KEYNOTE-012 study evaluated the safety and the efficacy of pembrolizumab for the treatment of HNSCC after long-term follow-up.

METHODS: Multi-centre, non-randomised trial included two HNSCC cohorts (initial and expansion) in which 192 patients were eligible. Patients received pembrolizumab $10 \mathrm{mg} / \mathrm{kg}$ every 2 weeks (initial cohort; $N=60$ ) or 200 mg every 3 weeks (expansion cohort; $N=132$ ). Co-primary endpoints were safety and overall response rate (ORR; RECIST v1.1; central imaging vendor review). RESULTS: Median follow-up was 9 months (range, 0.2-32). Treatment-related adverse events (AEs) of any grade and grade 3/4 occurred in $123(64 \%)$ and 24 (13\%) patients, respectively. No deaths were attributed to treatment-related AEs. ORR was 18\% (34/192; $95 \% \mathrm{Cl}, 13-24 \%)$. Median response duration was not reached (range, $2+$ to $30+$ months); $85 \%$ of responses lasted $\geq 6$ months. Overall survival at 12 months was $38 \%$.

CONCLUSIONS: Some patients received 2 years of treatment and the responses were ongoing for more than 30 months; the durable anti-tumour activity and tolerable safety profile, observed with long-term follow-up, support the use of pembrolizumab as a treatment for recurrent/metastatic HNSCC.

British Journal of Cancer (2018) 119:153-159; https://doi.org/10.1038/s41416-018-0131-9

\section{INTRODUCTION}

More than 500,000 new cases of head and neck squamous cell carcinoma (HNSCC) are diagnosed each year. ${ }^{1}$ Most patients present with locally advanced disease, which is most often managed using a multi-method approach that combines surgery, chemotherapy, and radiation therapy. ${ }^{2}$ With disease recurrence or metastatic disease, the standard first-line treatment is the combination of cetuximab, platinum, and fluorouracil (i.e., EXTREME regimen). ${ }^{3}$ Historically, treatment options were limited for patients with advanced HNSCC that progressed after first-line therapy ${ }_{i}^{3}$ however, recent results from clinical trials with immune checkpoint inhibitors have shown promising activity for secondline therapy. ${ }^{4-7}$

The programmed death 1 (PD-1) pathway is an important immune checkpoint exploited by immunosuppressive cancers including HNSCC to avoid immune detection. ${ }^{8}$ Binding of PD- 1 by either of its ligands, PD-L1 or PD-L2, suppresses the activation of effector T cells. ${ }^{9-11}$ Although this interaction functions to protect against excessive inflammation under normal conditions, it is hypothesised that upregulation of the PD-1 pathway allows cancer cells to develop adaptive immune resistance. ${ }^{12}$ Both PD-L1 and PD-L2 expression have been reported in HNSCC, ${ }^{13,14}$ and the PD-1 pathway has been established as an effective target in HNSCC.

Pembrolizumab, an anti-PD-1 antibody, has demonstrated robust anti-tumour activity and a manageable safety profile in multiple tumour types, and is currently approved in more than 60 countries for one or more advanced malignancies. ${ }^{15}$ Based on the safety and efficacy observed in patients with HNSCC enrolled in the phase lb KEYNOTE-012 trial (clinicaltrials.gov identifier: NCT01848834), pembrolizumab was approved by the US Food and Drug Administration for recurrent or metastatic HNSCC that has progressed on or after platinum-containing chemotherapy. ${ }^{15}$ Specifically, the KEYNOTE-012 trial enrolled two HNSCC cohorts: an initial cohort $(N=60)$ and an expansion cohort $(N=132)$. After

\footnotetext{
${ }^{1}$ Fox Chase Cancer Center, Philadelphia, PA, USA; ${ }^{2}$ University of Chicago, Chicago, IL, USA; ${ }^{3} \mathrm{H}$. Lee Moffitt Cancer Center \& Research Institute, Tampa, FL, USA; ${ }^{4}$ Lineberge Comprehensive Cancer Center at the University of North Carolina, Chapel Hill, NC, USA; ${ }^{5}$ Sheba Medical Center, Ramat Gan, Israel; ${ }^{6}$ Yale Cancer Center, New Haven, CT, USA; ${ }^{7}$ National Cancer Center Hospital East, Chiba, Japan; ${ }^{8}$ Seoul National University Hospital, Seoul, Republic of Korea; ${ }^{9}$ Johns Hopkins University, Baltimore, MD, USA; ${ }^{10}$ Aichi Cancer Center Hospital, Nagoya, Japan; ${ }^{11}$ Sourasky Medical Center, Tel Aviv, Israel; ${ }^{12}$ Yonsei Cancer Center, Yonsei University College of Medicine, Seoul, Republic of Korea; ${ }^{13}$ National Taiwan University Hospital, Taipei, Taiwan; ${ }^{14}$ Merck \& Co., Inc, Kenilworth, NJ, USA; ${ }^{15}$ University of Washington, Seattle, WA, USA; ${ }^{16}$ Dana-Farber Cancer Institute, Boston, MA, USA;

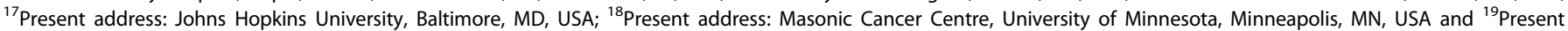
address: Yale Cancer Center, New Haven, CT, USA

Correspondence: Ranee Mehra (rmehra1@jhmi.edu)
}

Received: 30 November 2017 Revised: 26 April 2018 Accepted: 10 May 2018 Published online: 29 June 2018 
154

a median follow-up of 14 months (interquartile range [IQR], 4-14 months), the confirmed overall response rate (ORR) in the initial cohort was $18 \%(95 \% \mathrm{Cl}, 8-32 \%)$; responses lasted a median of 53 weeks $\left(95 \% \mathrm{Cl}, 13\right.$ weeks to not reached). ${ }^{6}$ An ORR of $18 \%$ $(95 \% \mathrm{Cl}, 12-26 \%)$ was also reported in the expansion cohort after a median follow-up of 9 months (IQR, 3-11 months); the median duration of response in this cohort was not reached at the time of reporting. ${ }^{5}$ Pembrolizumab was well tolerated in both cohorts: $17 \%$ and $9 \%$ of patients experienced grade 3/4 treatment-related adverse events (AEs) in the initial and the expansion cohorts, respectively.

Herein we report long-term results of patients in the two HNSCC cohorts of the multi-centre, non-randomised, phase Ib KEYNOTE012 trial, which investigated the safety and the efficacy of pembrolizumab in patients with advanced solid tumours., Because the frequency of response was similar across the two cohorts, data from patients in the initial and expansion cohorts were pooled for these analyses.

\section{METHODS}

Patients

Detailed eligibility criteria for the individual HNSCC cohorts have been published. ${ }^{5,6}$ Key inclusion criteria applicable to both cohorts included $\geq 18$ years of age; histologically or cytologically confirmed HNSCC; recurrent, metastatic, or persistent disease; measurable disease as per the Response Evaluation Criteria in Solid Tumors, version 1.1 (RECIST v1.1); and Eastern Cooperative Oncology Group performance status (ECOG PS), 0 or 1 . Only the initial cohort required evidence of PD-L1 expression. There was no limit to the number of prior therapies; however, prior treatment with immune checkpoint inhibitors was not allowed. Prior immunosuppressive therapy, chemotherapy, and therapy with anti-cancer monoclonal antibodies had to be concluded within 7 days, 2 weeks, and 4 weeks, respectively, before the start of the study treatment. The original studies were conducted in accordance with Good Clinical Practice guidelines and the Declaration of Helsinki, and the study protocol was approved by the institutional review boards or ethics committees of all participating sites. All patients provided written informed consent before study entry.

\section{Study design}

Pembrolizumab dose and administration schedule differed between the HNSCC cohorts. ${ }^{5,6}$ Patients in the initial cohort received pembrolizumab $10 \mathrm{mg} / \mathrm{kg}$ every 2 weeks; patients in the expansion cohort received pembrolizumab $200 \mathrm{mg}$ every 3 weeks. A lower dose and less frequent administration schedule was chosen for the expansion cohort based on data from the other pembrolizumab trials and pharmacodynamic modeling, which indicated that a lower dose and less frequent administration schedule were sufficient for target engagement and clinical activity. ${ }^{16,17}$ For both cohorts, treatment continued until confirmed disease progression, unacceptable toxicity, investigator's or patient's decision to withdraw, or completion of 24 months of treatment.

Tumour response was evaluated every 8 weeks using computed tomography or magnetic resonance imaging assessed per RECIST v1.1 by central imaging vendor review. Patients who experienced confirmed complete response (CR) could discontinue pembrolizumab if they received at least 24 weeks of treatment. If imaging indicated disease progression, the progression was to be substantiated by subsequent imaging performed no sooner than 4 weeks later. Clinically stable patients could remain on treatment during that time. If subsequent imaging indicated a reduction in tumour burden from what was seen with the initial imaging, the patient could continue treatment as scheduled.

AEs were monitored throughout the trial and for 30 days after the end of the treatment. Serious AEs and immune-mediated AEs
(imAE), defined as events with potential drug-related immunologic causes, regardless of attribution by the investigator, were monitored for 90 days after the treatment was ended. AEs were graded using the National Cancer Institute Common Terminology Criteria for Adverse Events, version 4.0. Pembrolizumab was withheld for most grade 3 treatment-related AEs, until toxicity was resolved to grade $0 / 1$. If toxicity did not resolve within 12 weeks of the last pembrolizumab dose, the treatment was discontinued. Treatment was also discontinued for grade 4 treatment-related $\mathrm{AEs}$ and for recurrent grade 3 treatment-related AEs.

The co-primary endpoints were safety and ORR (RECIST v1.1, central imaging vendor review). Secondary endpoints included ORR (RECIST v1.1, investigator review), ORR (RECIST v1.1, central imaging vendor review) in patients previously treated with cetuximab and platinum, progression-free survival (PFS), overall survival (OS), and duration of response (DOR).

Tumour analysis

Patients were required to provide the archival tissue samples or the newly obtained core or excisional tumour biopsy samples for human papillomavirus (HPV) and biomarker analyses. Patients were eligible, regardless of the HPV status. Patients were classified as having HPV-associated disease if the primary location of their tumour was in the oropharynx and the site investigator considered the tumour to be HPV positive. HPV-negative tumours determined by the individual study site and/or patients with primary tumour locations outside of the oropharynx were classified as non-HPV-associated disease.

PD-L1 expression status during screening was determined using a prototype PD-L1 immunohistochemical assay ${ }^{18}$ performed at a laboratory site (QualTek) accredited by the College of American Pathologists and Clinical Laboratory Improvement Amendments, and used commercially available reagents from the EnVision FLEX + HRP-Polymer kit (DAKO K8012; Agilent Technologies) and the anti-PD-L1 (clone 22C3) antibody (Merck \& Co., Inc.). Detailed methods for this assay have been described elsewhere. ${ }^{18}$

In a separate analysis of PD-L1 expression and anti-tumour response, PD-L1 expression was retrospectively evaluated using an investigational version of the PD-L1 IHC 22C3 pharmDx assay (Agilent Technologies). The staining protocol was performed according to the instructions of the commercial assay. ${ }^{19,20}$ The expression was scored using two methods: tumour proportion score (TPS) and combined positive score (CPS). TPS was defined as the percentage of tumour cells with membranous PD-L1 expression. CPS was defined the number of PD-L1-positive cells [tumour cells, lymphocytes, and macrophages] divided by the total number of tumour cells times 100 . Both scores ranged from 0 to 100; a cutoff of $\geq 1$ was used to define the PD-L1 expression.

Similarly, PD-L2 expression was retrospectively determined by immunohistochemistry using the anti-PD-L2 (clone 3G2) antibody (Merck \& Co., Inc.). PD-L2 expression was scored by determining the percentage of PD-L2-positive cells (tumour cells, macrophages, lymphocyte) over the total tumour cells. Scores ranged from 0 to $100 \%$; a $1 \%$ cut-off was used to define the PD-L2 expression.

\section{Statistical analysis}

In this pooled analysis, the efficacy and the safety were assessed in all patients with HNSCC who received at least one dose of pembrolizumab (all-patients-as-treated population). Efficacy endpoints were also analysed by subgroups based on the HPV status, biomarker expression, and prior therapies (platinum, platinum, and cetuximab [treatments could be concurrent or subsequent]).

ORR was defined as the proportion of patients in the analysis population who experienced confirmed $C R$ or partial response (PR). The response rates, point estimates, and $95 \% \mathrm{Cl}$ were determined using the exact binomial distribution. PFS was defined as the time from the first dose to the first instance of documented 
Efficacy and safety of pembrolizumab in recurrent...

R Mehra et al.

disease progression or death from any cause, whichever occurred first. OS was defined as the time from the first dose to death. DOR was defined as the time from the first confirmed response to disease progression. Kaplan-Meier statistics were used to estimate PFS, OS, and DOR. Patients with missing response data were considered non-responders; non-responders were excluded from the DOR analyses. Patients with missing survival data were censored at their last assessment.

Logistic (ORR) or Cox (PFS and OS) proportional hazards regression one-sided testing was performed to assess the relationship between efficacy and PD-L1 or PD-L2 expression.

\section{RESULTS}

Patients

A total of 192 patients with HNSCC from 16 centres in five countries were enrolled and received at least one dose of pembrolizumab, including 60 patients in the initial cohort and 132 patients in the expansion cohort. First patient was enrolled in 7 June, 2013, and the last patient was enrolled in 8 October, 2014. Therefore, the data cut-off for the current analysis (26 April, 2016) was more than 18 months after the last patient started the study.

Patient median age was 60 years (range, 20-84 years); $83 \%$ were men (Table 1 ). The majority of the patients were heavily pretreated: $74 \%$ received at least two prior lines of systemic therapy. Specifically, $91 \%$ of patients had received a platinum-based regimen, and $57 \%$ had received prior platinum and prior cetuximab treatment. Seventy-seven percent of patients had non-HPV-associated disease and $23 \%$ had HPV-associated disease.

The median follow-up, as of 26 April, 2016, was 9 months (range, 0.2-32 months). By that time, 168 (88\%) patients had discontinued the treatment, most commonly for progressive disease $(n=124)$ or an $\mathrm{AE}(n=23), 18(9 \%)$ patients remained on the treatment, and $6(3 \%)$ patients had completed 2 years of pembrolizumab (Fig. 1).

Safety

At data cut-off, the median time on pembrolizumab was 14 weeks (range, 0.1-107 weeks). Treatment-related AEs occurred in $64 \%$ $(n=123)$ of patients (Table 2). Thirteen percent $(n=24)$ experienced a treatment-related $A E$ of grade $3 / 4$ severity; increases in the alanine aminotransferase and the aspartate aminotransferase levels were the only grade 3/4 treatment-related AEs that occurred in more than two patients. There were 12 discontinuations ( $6 \%$ of patients) and zero deaths attributed to treatment-related AEs. Immune-mediated adverse events (imAEs) and infusion reactions, regardless of the attribution by the investigator, occurred in $24 \%$ $(n=46)$ of patients; the only imAEs that occurred in more than two patients were hypothyroidism (grade $1 / 2, n=26$; grade 3 , $n=2$ ), pneumonitis (grade $1 / 2, n=3$; grade $3, n=2$ ), adrenal insufficiency (grade $1 / 2, n=2$ ), and thyroiditis (grade $1 / 2, n=3$ ). All patients experiencing hypothyroidism received prior radiation. One grade $4 \mathrm{imAE}$ of diabetic ketoacidosis was reported, as was one grade $3 \mathrm{imAE}$ of each of the following: type 1 diabetes mellitus, decubitus ulcer, papule, rash, colitis, drug-induced liver injury, and macular rash. Two treatment-related cardiac events were reported in the same patient (atrial fibrillation and congestive heart failure; both grade 3 ) (Table 2).

\section{Efficacy}

ORR across all patients was $18 \%(95 \% \mathrm{Cl}, 13-24 \%)$ (Table 3). Eight (4\%) patients experienced CR and $26(14 \%)$ patients experienced PR. Another 33 (17\%) patients experienced stable disease (SD) and $93(48 \%)$ patients had progressive disease as the best response. Clinical benefit rate, defined as the proportion of patients experiencing $C R, P R$, or SD for $\geq 6$ months, was $20 \%(95 \% \mathrm{Cl}$, $15-27 \%)$. Similar response rates were reported when analyses
Table 1. Baseline demographics and characteristics (all-patients-astreated population)

\begin{tabular}{|c|c|}
\hline Characteristic & $N=192$ \\
\hline Age, median (range) (y) & $60(20-84)$ \\
\hline Male & $159(83)$ \\
\hline \multicolumn{2}{|l|}{ Race } \\
\hline White & $147(77)$ \\
\hline Asian & $29(15)$ \\
\hline Other & $16(8)$ \\
\hline \multicolumn{2}{|l|}{ ECOG performance status } \\
\hline 0 & $57(30)$ \\
\hline 1 & $135(70)$ \\
\hline \multicolumn{2}{|l|}{ Smoking status } \\
\hline Current/former & $140(73)$ \\
\hline Never & $52(27)$ \\
\hline \multicolumn{2}{|l|}{ HPV status } \\
\hline Associated & $45(23)$ \\
\hline Not associated & $147(77)$ \\
\hline Prior radiation & $146(76)$ \\
\hline Prior surgery & $129(67)$ \\
\hline Prior adjuvant and/or neoadjuvant therapy, ${ }^{\mathrm{a}} n$ & $90(47)$ \\
\hline No. of prior lines of systemic therapies, median (range), $n$ & $2(0-7)$ \\
\hline \multicolumn{2}{|l|}{ No. of previous lines of systemic therapy ${ }^{b}$} \\
\hline 1 & $47(24)$ \\
\hline 2 & $56(29)$ \\
\hline$\geq 3$ & $86(45)$ \\
\hline Prior platinum therapy & $174(91)$ \\
\hline Prior platinum and cetuximab therapy & $110(57)$ \\
\hline \multicolumn{2}{|l|}{ Metastatic stage } \\
\hline $\mathrm{MX}$ & $1(<1)$ \\
\hline Mo & $26(14)$ \\
\hline M1 & $165(86)$ \\
\hline
\end{tabular}

The data are no. (\%) unless otherwise stated. ECOG Eastern Cooperative

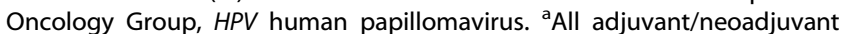
therapies were chemotherapies. ${ }^{\mathrm{b}}$ Three patients had 0 lines of systemic therapy

were restricted to patients whose disease progressed after prior platinum therapy $(17 \%[95 \% \mathrm{Cl}, 12-23 \%])$, or prior platinum and prior cetuximab therapy $(15 \%[95 \% \mathrm{Cl}, 9-23 \%])$ (Supplemental Table 1). ORR was $24 \%(95 \% \mathrm{Cl}, 13-40 \%)$ among patients with HPV-associated disease and $16 \%(95 \% \mathrm{Cl}, 10-23)$ among those with non-HPV-associated disease (Table 3). Decrease in the target lesion size from baseline was observed in $60 \%$ of all patients, including $57 \%$ with HPV-associated disease and $62 \%$ with nonHPV-associated disease (Fig. 2a).

Among the 34 responders, the median time to response was 2 months (range, 2-17 months) (Fig. 2b). Median DOR was not reached (range, $2+$ to $30+$ months) (Fig. 2c; Supplemental Table 2). Based on Kaplan-Meier estimates, $85 \%$ of responses lasted $\geq 6$ months and $71 \%$ of responses lasted $\geq 12$ months, and $65 \%$ of responses were ongoing at the data cut-off with three lasting at least 2 years. Among those patients who experienced response, 15 were still receiving pembrolizumab, 2 discontinued pembrolizumab after experiencing $C R$, and 5 completed the study after receiving pembrolizumab for 2 years.

Median PFS was 2.1 months (95\% Cl, 1.9-2.1 months) (Fig. 3a). PFS rates at 6 and 12 months were $25 \%$ and $17 \%$, respectively. Median OS was 8 months (95\% Cl, 6-10 months) (Fig. 3b). 


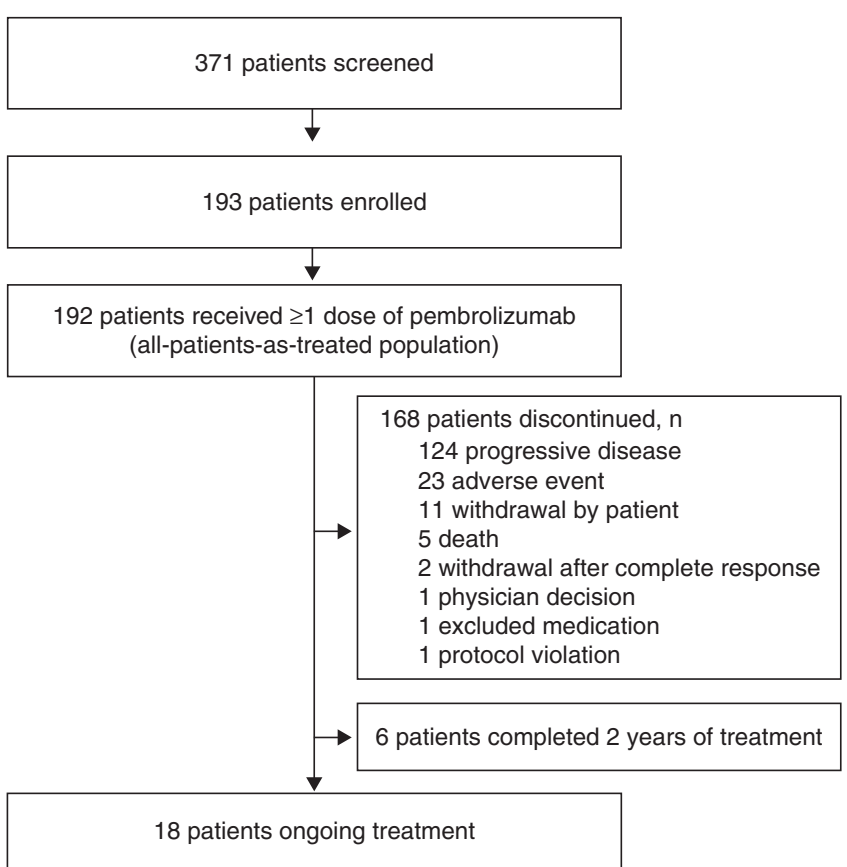

Fig. 1 Patient disposition

The 6 -month OS rate was $58 \%$, and the 12 -month OS rate was $38 \%$.

Biomarker analysis

Efficacy and PD-L1 expression data were available for 188 patients. When PD-L1 expression was determined using TPS, 123 (65\%) patients had PD-L1-expressing tumours, whereas 65 (35\%) patients had tumours that did not express PD-L1. When PD-L1 expression was determined using CPS, 152 (81\%) patients had PD-L1expressing tumours and 36 (19\%) patients had non-PD-L1expressing tumours. Significantly, higher response rates were observed in patients with vs. without PD-L1 expression using CPS (21 vs. 6\%; one-sided $P=0.023$ ), but not TPS (Supplemental Table 3). Similarly, the median $(95 \% \mathrm{Cl})$ PFS rates were significantly different using CPS (PD-L1-expressing, 2.1 months [1.9-3.2 months]; non-PDL1-expressing, 2.0 months [1.7-2.2 months]; one-sided $P=0.026$ ), but not TPS (Supplemental Fig. 1A). The median $(95 \% \mathrm{Cl})$ OS rates were also significantly different when CPS was used (PD-L1expressing, 10 months [9-13 months]; non-PD-L1-expressing, 5 months [3-8 months]; one-sided $P=0.008$ ) (Supplemental Fig. 1B).

PD-L2 expression data were available for 172 patients; 111 (65\%) patients had PD-L2-expressing tumours and 61 (35\%) patients had non-PD-L2-expressing tumours. A significant positive correlation was observed between PD-L1 and PD-L2 expression (one-sided $P<0.001$ ). A significantly higher response rate was seen in patients with vs. without tumours that expressed PD-L2 (23\% vs. $10 \%$; one-sided $P=0.022$ ) (Supplemental Table 3). Additionally, higher ORR was noted in PD-L1-expressing tumours that also expressed PD-L2 $(n=108)$, compared with those that did not express PD-L2 ( $n=39)$ ( $23 \%$ vs. $10 \%)$

\section{DISCUSSION}

The last agent to receive approval from the US Food and Drug Administration as second-line therapy for HNSCC before 2016 was cetuximab in 2006 , with a reported ORR of $13 \%$ (95\% Cl, 7-21\%). ${ }^{21}$ In the subsequent decade, there has been little further advancement with second-line options plagued by low response rates $(6-13 \%)$ and toxicity. ${ }^{22,23}$ Results presented herein after long-term follow-up confirm the anti-tumour activity and tolerability of

\begin{tabular}{|c|c|}
\hline Treatment-related adverse event & $\begin{array}{l}\text { Any grade occurring in } \geq 2 \% \text { of } \\
\text { patients (No. (\%)) }\end{array}$ \\
\hline Any & $123(64)$ \\
\hline Fatigue & $42(22)$ \\
\hline Hypothyroidism & $19(10)$ \\
\hline Rash & $18(9)$ \\
\hline Pruritus & $16(8)$ \\
\hline Appetite decrease & $16(8)$ \\
\hline Pyrexia & $12(6)$ \\
\hline Nausea & $11(6)$ \\
\hline Arthralgia & $10(5)$ \\
\hline Dry skin & $9(5)$ \\
\hline Weight decrease & $9(5)$ \\
\hline AST level increase & $6(3)$ \\
\hline Facial swelling & $6(3)$ \\
\hline Anaemia & $8(4)$ \\
\hline ALT level increase & $5(3)$ \\
\hline Myalgia & $5(3)$ \\
\hline Diarrhoea & $5(3)$ \\
\hline Pneumonitis & $5(3)$ \\
\hline Stomatitis & $4(2)$ \\
\hline Vomiting & $4(2)$ \\
\hline Chills & $4(2)$ \\
\hline Blood TSH level increase & $4(2)$ \\
\hline Hyponatremia & $4(2)$ \\
\hline \multirow[t]{2}{*}{ Maculopapular rash } & $4(2)$ \\
\hline & $\begin{array}{l}\text { Grade } 3 / 4 \text { occurring in } \geq 2 \text { patients } \\
\text { (No. }(\%))\end{array}$ \\
\hline Any & $24(13)$ \\
\hline ALT level increase & $3(2)$ \\
\hline AST level increase & $3(2)$ \\
\hline Hypothyroidism & $2(1)$ \\
\hline Fatigue & $2(1)$ \\
\hline Appetite decrease & $2(1)$ \\
\hline Hyponatremia & $2(1)$ \\
\hline Pneumonitis & $2(1)$ \\
\hline \multirow[t]{2}{*}{ Facial swelling } & $2(1)$ \\
\hline & $\begin{array}{l}\text { Rare events of interest } \\
\text { (No. (\%) [grade]) }\end{array}$ \\
\hline \multicolumn{2}{|l|}{ Immune-mediated } \\
\hline Adrenal insufficiency & $2(1)[1,2]$ \\
\hline Colitis & 1 (1) [3] \\
\hline Diabetic ketoacidosis & $1(1)[4]$ \\
\hline Type 1 diabetes mellitus & $1(1)[3]$ \\
\hline \multicolumn{2}{|l|}{ Cardiac } \\
\hline Atrial fibrillation & $1(1)[3]$ \\
\hline Congestive heart failure & 1 (1) [3] \\
\hline
\end{tabular}

ALT alanine aminotransferase, AST aspartate aminotransferase, $T S H$ thyroid stimulating hormone

pembrolizumab in the heavily pre-treated HNSCC patient population enrolled in KEYNOTE-012. This data set represents, to our knowledge, the longest follow-up period of patients with HNSCC who were treated with a PD-1 inhibitor and highlights long 
Table 3. Tumour response to pembrolizumab as per RECIST v1.1 by central imaging vendor review (all-patients-as-treated population; $N=192)$

\begin{tabular}{|c|c|c|c|c|c|c|}
\hline & \multicolumn{2}{|c|}{ AllN=192 } & \multicolumn{2}{|c|}{$\begin{array}{l}\text { HPV associated } \\
n=45\end{array}$} & \multicolumn{2}{|c|}{$\begin{array}{l}\text { Non-HPV } \\
\text { associated } \\
n=147\end{array}$} \\
\hline & No. & $\%(95 \% \mathrm{Cl})$ & No. & $\%(95 \% \mathrm{Cl})$ & No. & $\%(95 \% \mathrm{Cl})$ \\
\hline $\begin{array}{l}\text { Overall response } \\
\text { rate }\end{array}$ & 34 & $18(13-24)$ & 11 & $24(13-40)$ & 23 & $16(10-23)$ \\
\hline $\begin{array}{l}\text { Complete } \\
\text { response }\end{array}$ & 8 & $4(2$ & 4 & 9( & 4 & 31 \\
\hline Partial response & 26 & $14(9-19)$ & 7 & $16(7-30)$ & 19 & $13(8-19)$ \\
\hline Stable disease & 33 & $17(12-23)$ & 7 & $16(7-30)$ & 26 & $18(12-25)$ \\
\hline $\begin{array}{l}\text { Progressive } \\
\text { disease }\end{array}$ & 93 & $48(41-56)$ & 19 & $42(28-58)$ & 74 & $50(42-59)$ \\
\hline Non-CR/Non-PD & 7 & $4(2-7)$ & 1 & $2(0.1-12)$ & 6 & $4(2-9)$ \\
\hline No assessment & 21 & $11(7-16)$ & 6 & $13(5-27)$ & 15 & $10(6-16)$ \\
\hline Not evaluable & 4 & $2(0.6-5)$ & 1 & $2(0.1-12)$ & 3 & $2(0.4-6)$ \\
\hline \multicolumn{7}{|c|}{$\begin{array}{l}\text { Only confirmed responses are included. } C R \text { complete response, PD } \\
\text { progressive disease, RECIST Response Evaluation Criteria in Solid Tumours. } \\
\text { No assessment: patient discontinued before the first imaging assessment } \\
\text { (reasons: progressive disease }[n=12] \text {; adverse event }[n=3] \text {; withdrawal by } \\
\text { patient }[n=3] \text {; death }[n=2] \text {; protocol violation }[n=1] \text { ). Not evaluable: } \\
\text { patient had post baseline imaging, but images were not of sufficient } \\
\text { quality to determine response }\end{array}$} \\
\hline
\end{tabular}

durability of responses achieved with some patients. Furthermore, the impact on survival seems to be significant; with a 38\% 12month OS rate, it is likely that a larger fraction of patients than just those who experienced the response will benefit from the treatment. Although the last patient with HNSCC was enrolled more than 18 months before these analyses were performed, the median DOR was not reached, and $65 \%$ of responses were ongoing, with some lasting for more than 30 months. Consistent with the reports from the individual cohorts, $18 \%$ of patients experienced $\mathrm{CR}$ or PR, and responses were observed, regardless of the HPV status. ${ }^{5,6}$ Despite the long-term treatment, pembrolizumab was well tolerated. The safety profile of pembrolizumab was consistent with profiles reported in other tumour types ${ }^{16,24-27}$ and no new safety risks were identified.

Although this trial did not mandate specific prior therapies, patients were heavily pre-treated. Importantly, $17 \%$ of patients treated previously with platinum and $15 \%$ treated previously with platinum and cetuximab treatment responded to pembrolizumab. This aligns with the $16 \%$ ORR in patients with HNSCC that progressed after platinum and cetuximab who were treated with pembrolizumab in the phase II KEYNOTE-055 trial. ${ }^{7}$ Another PD-1 inhibitor, nivolumab, was studied in CheckMate-141, in which patients were randomly assigned to receive nivolumab $3 \mathrm{mg} / \mathrm{kg}$ every 2 weeks, compared with the standard of care. The investigators reported an ORR of $13 \%$ and OS of 7.5 months. In a subset analysis, the HR was reported to be 0.55 among patients with PD-L1 expression of $\geq 1 \%{ }^{4}$ This enrichment for response based on PD-L1 status is consistent with the current study, although different assays and expression analyses were used.
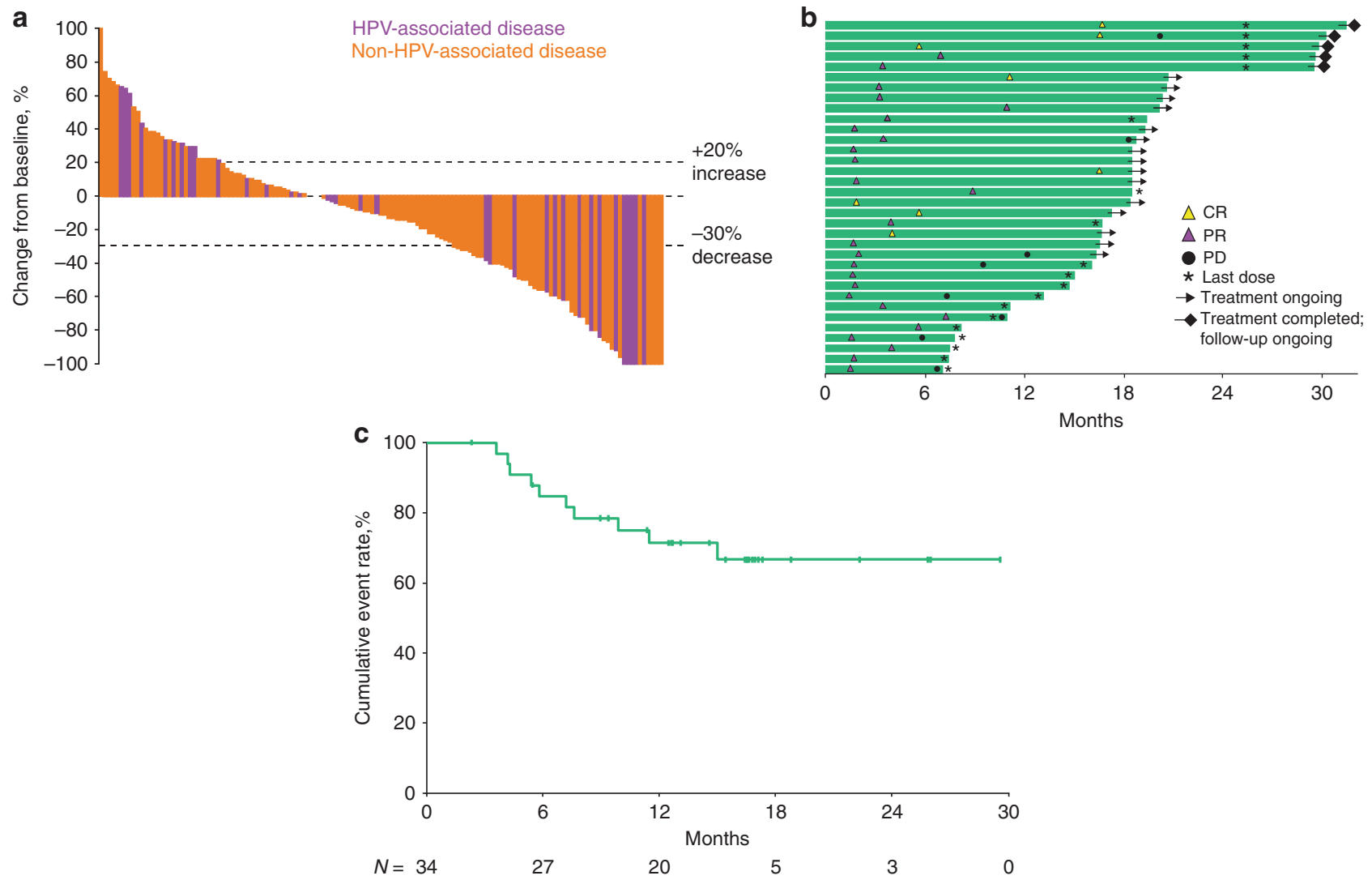

Fig. 2 Tumour response to pembrolizumab according to RECIST v1.1 by central imaging vendor review. a Best percentage change from baseline in target lesions $(n=139)$. Includes patients who had measurable disease at baseline and at least one post baseline scan. $\mathbf{b}$ Treatment exposure and duration of response in patients achieving partial responses or complete responses $(n=34)$. c Kaplan-Meier estimate of the duration of response in patients achieving partial responses or complete responses. RECIST Response Evaluation Criteria in Solid Tumors 

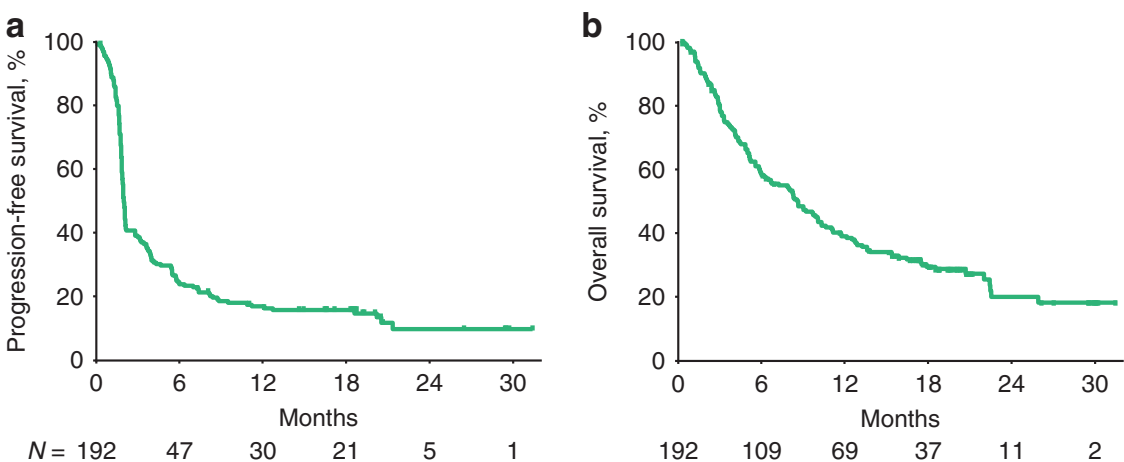

Fig. 3 Survival in patients treated with pembrolizumab. Kaplan-Meier estimates of a progression-free survival per RECIST v1.1 by central imaging vendor review and $\mathbf{b}$ overall survival (all-patients-as-treated population). RECIST Response Evaluation Criteria in Solid Tumors

As reported with individual cohorts, significant association between PD-L1 expression and response was observed when the analysis included the expression in both tumour and immune cells (CPS). ${ }^{5,6}$ Adding to these findings, we noted similar correlations of PD-L1 expression with OS and PFS using CPS. Association of PD-L1 expression per CPS and response to pembrolizumab was also reported in patients with advanced HNSCC in KEYNOTE-055. ${ }^{7}$ In addition, we demonstrated a significant association between PDL1 and PD-L2 expression and found that PD-L2-expressing tumours were more likely to respond to pembrolizumab than non-PD-L2-expressing tumours. Nonetheless, patients without expression of either biomarker also responded to pembrolizumab at a clinically meaningful rate (9\%). Therefore, although the use of PD-L1 and PD-L2 expression as biomarkers may enrich the response, patients whose tumours do not express these biomarkers may still respond to pembrolizumab.

These findings raise the questions of whether and how to use the biomarkers for patient selection. Currently, pembrolizumab is approved for use in recurrent or metastatic HNSCC after platinum therapy, regardless of the PD-L1 expression. Studies have already suggested the potential usefulness of an immune-related gene signature to predict the response to the PD-1 checkpoint blockade ${ }^{28}$; however, additional studies are necessary to determine whether a multigene profile or other biomarker can aid in the treatment decisions.

In conclusion, pembrolizumab exhibited durable anti-tumour activity, high survival rates, and acceptable safety in patients with heavily pre-treated advanced HNSCC. These data demonstrate that a subset of patients will receive long-term benefit from treatment with pembrolizumab, a paradigm rarely observed with existing cytotoxic or targeted therapies for recurrent or metastatic HNSCC. Combination studies of pembrolizumab with additional immunetargeting agents and with radiation therapy are under way.

\section{ACKNOWLEDGEMENTS}

We thank the patients and their families, and caregivers for participating in the study. We also thank Jennifer Yearley of Merck \& Co., Inc., Kenilworth, NJ, USA, for her contributions to this research. Medical writing and/or editorial assistance was provided by Matthew Grzywacz, PhD, Dana Francis, PhD, and the ApotheCom pembrolizumab team (Yardley, PA, USA). This assistance was funded by Merck \& Co., Inc., Kenilworth, NJ, USA.

\section{AUTHORS CONTRIBUTIONS}

The study was designed and funded by Merck \& Co., Inc., Kenilworth, NJ, USA. R.M., T. Y.S., J.W., I.G., J.P.E., B.B., M.T., B.K., H.K., M.K., H.C.C., C.C.L., A.R., K.P., L.Q.W., and R.H. collected the data. R.M., J.W., B.B., H.C.C., D.A.-G., K.P., J.C., L.Q.W., and R.H. analyzed the data. R.M., T.Y.S., J.W., I.G., B.B., M.T., B.K., H.K., H.C.C., C.C.L., K.P., J.C., L.Q.W., and R. H. contributed to the interpretation of the results. R.M., M.T., and K.P. contributed to drafting of the manuscript.

\section{ADDITIONAL INFORMATION}

Supplementary information is available for this paper at https://doi.org/10.1038/ s41416-018-0131-9.

Consent for publication: All authors had full access to all the data in the study, contributed to reviewing and revising the manuscript, and had final responsibility for the decision to submit for publication.

Competing interests: T.Y.S., J.W., B.B., and H.K. have received research funding from Merck \& Co., Inc. M.T. has received personal fees from Merck Sharp \& Dohme. D.A.-G., A.R., K.P., and J.C. are employees Merck Sharp \& Dohme Corp., a subsidiary of Merck \& Co., Inc., Kenilworth, NJ, USA. L.Q.C. has served as an advisor for and has received research from Merck \& Co., Inc. R.H. has received research funding from Merck \& Co., Inc., Bristol-Myers Squibb, Pfizer, and Astra Zeneca, and has served as a consultant for Merck \& Co., Inc., Bristol-Myers Squibb, Pfizer, Celgene, Astra Zeneca, and Eisai. The remaining authors declare no competing interests.

Ethical approval and consent to participate: The original studies were conducted in accordance with Good Clinical Practice guidelines and the Declaration of Helsinki, and the study protocol was approved by institutional review boards or ethics committees of all participating sites.

Availability of data and material: Merck \& Co., Inc.'s data sharing policy including restrictions is available at http://engagezone.merck.com/ds_documentation.php. Requests for access to the clinical study data can be submitted through the EngageZone site or via email to dataaccess@merck.com.

Note: This work is published under the standard license to publish agreement. After 12 months the work will become freely available and the license terms will switch to a Creative Commons Attribution 4.0 International licence (CC BY 4.0).

Publisher's note: Springer Nature remains neutral with regard to jurisdictional claims in published maps and institutional affiliations.

\section{REFERENCES}

1. Ferlay, J. et al. Cancer incidence and mortality worldwide: sources, methods and major patterns in GLOBOCAN 2012. Int. J. Cancer 136, E359-E386 (2015).

2. Pignon, J. P., le, M. A., Maillard, E. \& Bourhis, J. Meta-analysis of chemotherapy in head and neck cancer (MACH-NC): an update on 93 randomised trials and 17,346 patients. Radiother. Oncol. 92, 4-14 (2009).

3. National Comprehensive Cancer Network. NCCN clinical practice guidelines (NCCN Guidelines') in oncology: head and neck cancers. Version 1.2018. https:// www.nccn.org/professionals/physician_gls/pdf/head-and-neck.pdf. Accessed 19 Apr 2018 (2018).

4. Ferris, R. L. et al. Nivolumab for recurrent squamous-cell carcinoma of the head and neck. N. Engl. J. Med 375, 1856-1867 (2016).

5. Chow, L. Q. et al. Antitumor activity of pembrolizumab in biomarker-unselected patients with recurrent and/or metastatic head and neck squamous cell carcinoma: results from the phase Ib KEYNOTE-012 expansion cohort. J. Clin. Oncol. 34, 3838-3845 (2016).

6. Seiwert, T. Y. et al. Safety and clinical activity of pembrolizumab for treatment of recurrent or metastatic squamous cell carcinoma of the head and neck (KEYNOTE-012): an open-label, multicentre, phase 1b trial. Lancet Oncol. 17, 956-965 (2016). 
7. Bauml, J. et al. Preliminary results from KEYNOTE-055: pembrolizumab after platinum and cetuximab failure in head and neck squamous cell carcinoma (HNSCC). J. Clin. Oncol. 35, 1542-1549 (2016).

8. Pardoll, D. M. The blockade of immune checkpoints in cancer immunotherapy. Nat. Rev. Cancer 12, 252-264 (2012).

9. Blank, $C$. et al. PD-L1/B7H-1 inhibits the effector phase of tumor rejection by $T$ cell receptor (TCR) transgenic CD8 ${ }^{+}$T cells. Cancer Res. 64, 1140-1145 (2004).

10. Freeman, G. J. et al. Engagement of the PD-1 immunoinhibitory receptor by a novel B7 family member leads to negative regulation of lymphocyte activation. $J$. Exp. Med. 192, 1027-1034 (2000).

11. Latchman, Y. et al. PD-L2 is a second ligand for PD-1 and inhibits T cell activation. Nat. Rev. Immunol. 2, 261-268 (2001).

12. Iwai, Y. et al. Involvement of PD-L1 on tumor cells in the escape from host immune system and tumor immunotherapy by PD-L1 blockade. Proc. Natl Acad. Sci. USA 99, 12293-12297 (2002).

13. Strome, S. E. et al. B7-H1 blockade augments adoptive T-cell immunotherapy for squamous cell carcinoma. Cancer Res. 63, 6501-6505 (2003).

14. Tsushima, F. et al. Predominant expression of $\mathrm{B} 7-\mathrm{H} 1$ and its immunoregulatory roles in oral squamous cell carcinoma. Oral Oncol. 42, 268-274 (2006).

15. Merck \& Co. Inc. KEYTRUDA ${ }^{\circledast}$ (pembrolizumab) for Injection, for Intravenous Use KEYTRUDA ${ }^{\circledR}$ (Pembrolizumab) Injection, for Intravenous Use (Merck \& Co., Inc., Whitehouse Station, NJ, 2017).

16. Hamid, O. et al. Randomized comparison of two doses of the anti-PD-1 monoclonal antibody MK-3475 for ipilimumab-refractory (IPI-R) and IPI-naive (IPI-N) melanoma (MEL). J. Clin. Oncol. 32 (2014). 15_suppl. Abstract 3000.

17. Freshwater, T. et al. Evaluation of dosing strategy for pembrolizumab for oncology indications. J. Immunother. Cancer 5, 43 (2017).

18. Dolled-Filhart, $M$. et al. Development of a prototype immunohistochemistry assay to measure programmed death ligand-1 expression in tumor tissue. Arch. Pathol. Lab. Med. 140, 1259-1266 (2016).

19. Agilent Technologies. Agilent technologies receives expanded FDA approval for use of Dako PD-L1 IHC 22C3 pharmDx companion diagnostic in non-small cell lung cancer (NSCLC), press release, 24 October 2016, http://www.businesswire. com/news/home/20161024006586/en/Agilent-Technologies-Receives-ExpandedFDA-Approval-Dako (2016).

20. Dolled-Filhart, M. et al. Development of a companion diagnostic for pembrolizumab in non-small cell lung cancer using immunohistochemistry for programmed death ligand-1. Arch. Pathol. Lab. Med. 140, 1243-1249 (2016).

21. Vermorken, J. B. et al. Open-label, uncontrolled, multicenter phase II study to evaluate the efficacy and toxicity of cetuximab as a single agent in patients with recurrent and/or metastatic squamous cell carcinoma of the head and neck who failed to respond to platinum-based therapy. J. Clin. Oncol. 25, 2171-2177 (2007).

22. Machiels, J. P. et al. Afatinib versus methotrexate as second-line treatment in patients with recurrent or metastatic squamous-cell carcinoma of the head and neck progressing on or after platinum-based therapy (LUX-Head \& Neck 1): an open-label, randomised phase 3 trial. Lancet Oncol. 16, 583-594 (2015).

23. Machiels, J. P. et al. Zalutumumab plus best supportive care versus best supportive care alone in patients with recurrent or metastatic squamous-cell carcinoma of the head and neck after failure of platinum-based chemotherapy: an open-label, randomised phase 3 trial. Lancet Oncol. 12, 333-343 (2011).

24. Garon, E. B. et al. Pembrolizumab for the treatment of non-small-cell lung cancer N. Engl. J. Med. 372, 2018-2028 (2015).

25. Hamid, O. et al. Safety and tumor responses with lambrolizumab (anti-PD-1) in melanoma. N. Engl. J. Med. 369, 134-144 (2013).

26. Plimack, E. R. et al. Safety and activity of pembrolizumab in patients with locally advanced or metastatic urothelial cancer (KEYNOTE-012): a non-randomised, open-label, phase 1b study. Lancet Oncol. 18, 212-220 (2017).

27. Muro, K. et al. Pembrolizumab for patients with PD-L1-positive advanced gastric cancer (KEYNOTE-012): a multicentre, open-label, phase 1b trial. Lancet 17 717-726 (2016).

28. Ayers, M. et al. IFN-gamma-related mRNA profile predicts clinical response to PD1 blockade. J. Clin. Invest. 127, 2930-2940 (2017). 